\title{
Pitch Angle Control by Regenerative Air Brake for Electric Aircraft
}

\author{
Kentaro Yokota*a) $\quad$ Student Member, Hiroshi Fujimoto* Senior Member
}

\begin{abstract}
Conventional aircraft use mechanical air brakes to adjust their descent paths; however, the lift-drag ratio cannot be continuously controlled, which results in zigzag descent paths. Previous research has shown that the negative thrust produced by the windmilling propeller works as a substitute air brake called the regenerative air brake. This paper proposes a new pitch angle control method using the regenerative air brake. The proposed method includes thrust estimation, negative thrust control, airspeed estimation, and pitch angle control using the pitching-jerk-based disturbance observer. The effectiveness of the proposed method is verified via simulations and experiments in a wind tunnel.
\end{abstract}

Keywords: Electric aircraft, propeller dynamics, thrust estimation, thrust control, airspeed estimation, regeneration power, attitude control

\section{Introduction}

1.1 Electric Aircraft Owing to the increasing demand for personal and eco-friendly aviation, research and development in electric aircraft (EA) have attracted attention. Because EA are powered by electric motors, they have the following advantages from the control engineering viewpoint:

(1) Motor torque generation is 100 times faster than that of internal combustion engines ${ }^{(1)}$.

(2) Motor torque measurement is accurate ${ }^{(1)}$.

(3) Distributed installation (DEP: Distributed Electric Propulsion) and independent control of motors are easy ${ }^{(2)}$.

(4) Power regeneration is possible ${ }^{(3)}$

These advantages make EA safer, more efficient and environmental friendly.

The authors' research group has been studying new control methods for propeller-driven electric aircraft, proposed quick thrust and lift ${ }^{(4)(5)}$, and range extension systems ${ }^{(6)}$ by adopting the motion control theories developed in the automotive industry ${ }^{(7)}$.

1.2 Power Regeneration by Propellers Previous studies have shown that windmilling propellers produce negative thrust and torque, allowing the motor to generate electric power ${ }^{(3)(8) \wedge(11)}$. It is estimated that an EA can regenerate approximately $10 \%$ of its potential energy while descending ${ }^{(3)}$, and the energy generated during descent allows the EA to cruise about $60 \%$ of the descent distance ${ }^{(11)}$. Regeneration during descent reduces the batteries for descent, loiter, goaround, and taxiing.

1.3 Regenerative Air Brake Conventional aircraft use mechanical air brakes to adjust the descent path. However, they cannot be continuously controlled, making them difficult to operate even for a skilled pilot ${ }^{(8)}$. The airfield requests the top of descent (TOD) and the bottom of descent

\footnotetext{
a) Correspondence to: yokota.kentaro19@ae.k.u-tokyo.ac.jp

${ }^{*}$ The University of Tokyo

5-1-5, Kashiwanoha, Kashiwa, Chiba, 227-8561 Japan

Phone: +81-4-7136-3873
}

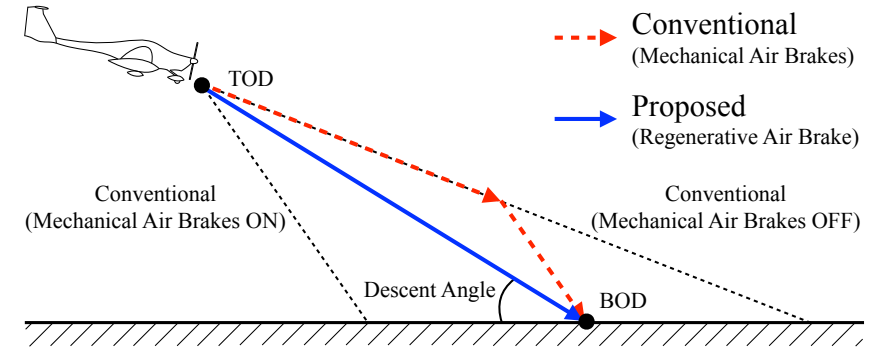

Fig. 1. Conventional and proposed descent paths.

(BOD), but because of this difficulty, the conventional aircraft descends in a zigzag path, as shown in Fig. 1.

The idea of the regenerative air brake suggests that the negative thrust of the windmilling propeller can substitute for conventional mechanical air brakes ${ }^{(1)}$. Compared to mechanical air brakes, the regenerative air brake requires no additional actuators and has better control performance. The regenerative air brake was first demonstrated in flight tests conducted by JAXA ${ }^{(10)}$. However, in this method, the controller only provided the input torque command on a feedforward basis, leading to poor robustness. In addition, the adjustment of the descent angle still depended on the ability of the pilot because the pilot could only change the input torque. To achieve better descent control performance, the propeller thrust should be controlled. Previous research on force control of propellers included thrust control for the $\mathrm{EA}^{(4)}$ and contact force control for drones ${ }^{(12)}$.

1.4 About this Study This study aims to realize a new attitude control utilizing the control capability of the regenerative air brake that achieves descent without mechanical air brakes. The proposed method comprises four steps.

Step 1 involves observer-based thrust estimator. Existing aircraft do not have force sensors on propellers, since adding such sensors degrades the stiffness of the thrusters. Therefore, the propeller thrust cannot be measured, and thus, must be estimated.

Step 2 involves negative thrust control. The thrust controller for the regenerative air brake was designed. 
Table 1. Parameter definitions.

\begin{tabular}{|c|c|c|}
\hline Symbol & Definition & Unit \\
\hline$\rho$ & Air density & $\mathrm{kg} \mathrm{m}^{-3}$ \\
\hline$V$ & Airspeed & $\mathrm{m} \mathrm{s}^{-1}$ \\
\hline$J_{\omega}$ & Inertia moment of propeller motor & $\mathrm{kg} \mathrm{m}^{2}$ \\
\hline$B_{\omega}$ & Viscosity coefficient of motor & $\mathrm{Nmsrad}^{-1}$ \\
\hline$T_{C}$ & Coulomb friction of motor & $\mathrm{Nm}$ \\
\hline$T$ & Input toque of motor & $\mathrm{Nm}$ \\
\hline$\beta$ & Propeller pitch angle & deg \\
\hline$D_{p}$ & Propeller diameter & $\mathrm{m}$ \\
\hline$C_{F}$ & Thrust coefficient of propeller & - \\
\hline$C_{Q}$ & Torque coefficient of propeller & - \\
\hline$F$ & Propeller thrust & $\mathrm{N}$ \\
\hline$Q$ & Counter torque of propeller & $\mathrm{Nm}$ \\
\hline$n$ & Rotational speed of propeller & $\mathrm{rps}$ \\
\hline$J$ & Advance ratio & - \\
\hline$m$ & Mass of aircraft & $\mathrm{kg}$ \\
\hline$S$ & Area of wing & $\mathrm{m}^{2}$ \\
\hline$C_{L}$ & Lift coefficient of wing & - \\
\hline$C_{D}$ & Drag coefficient of wing & - \\
\hline$L$ & Lift of aircraft & $\mathrm{N}$ \\
\hline$D$ & Drag of aircraft & $\mathrm{N}$ \\
\hline$\alpha$ & Perturbed angle of attack & $\mathrm{rad}$ \\
\hline$\alpha_{0}$ & Trim angle of attack & $\mathrm{rad}$ \\
\hline$q$ & Perturbed pitch rate & $\operatorname{rad~s}^{-1}$ \\
\hline$\theta$ & Perturbed pitch angle & $\mathrm{rad}, \mathrm{deg}$ \\
\hline$\Theta_{0}$ & Trim pitch angle of aircraft & $\mathrm{rad}, \mathrm{deg}$ \\
\hline$u_{x}$ & Perturbed $x$-axis velocity & $\mathrm{m} \mathrm{s}^{-1}$ \\
\hline$U_{x 0}$ & Trim $x$-axis velocity & $\mathrm{m} \mathrm{s}^{-1}$ \\
\hline$F_{0}$ & Trim thrust & $\mathrm{N}$ \\
\hline$\Delta F$ & Thrust change from trim & $\mathrm{N}$ \\
\hline$\delta_{\mathrm{ab}}$ & Deflection angle of air brakes & $\mathrm{rad}$ \\
\hline$\delta_{\mathrm{t}}$ & Throttle setting & - \\
\hline$X_{u_{X}}, X_{\alpha}, X_{q}$ & Stability derivatives for $x$-axis force & \\
\hline$Z_{u_{x}}, Z_{\alpha}, Z_{q}$ & Stability derivatives for $z$-axis force & \\
\hline$M_{u_{x}}, M_{\alpha}, M_{\dot{\alpha}}, M_{q}$ & Stability derivatives for pitching moment & \\
\hline$X_{\delta_{\mathrm{ab}}}, X_{\delta_{\mathrm{t}}}$ & Control derivatives for $x$-axis force & \\
\hline$Z_{\delta_{\mathrm{ab}}}, Z_{\delta_{\mathrm{t}}}$ & Control derivatives for $z$-axis force & \\
\hline$M_{\delta_{\mathrm{ab}}}, M_{\delta_{\mathrm{t}}}$ & Control derivatives for pitching moment & \\
\hline
\end{tabular}

Step 3 involves airspeed estimation. The regeneration conditions, such as the lower limit of the thrust, vary depending on the airspeed, but the Pitot tube has poor responsiveness. Airspeed must be estimated quickly.

Step 4 is the pitch angle control. The pitch angle controller is designed using Steps 1-3 and a pitching-jerk-based disturbance observer (PJBDOb).

The remainder of this paper is organized as follows. Section 2 describes the modeling of a single-motor EA. Section 3 describes the four steps of the proposed method. Finally, section 4 presents the simulation and experimental results.

Table 1 shows the definitions of the plant parameters in this study.

\section{Modeling of Single Motor Electric Aircraft}

In this section, a single motor EA is modeled.

2.1 Aircraft Dynamics Fig. 2(a) shows a view of the aircraft in a steady descent. The vector sum of all forces is zero because the aircraft is in equilibrium. Hence, in the direction of the descent,

$$
F_{0} \cos \alpha_{0}+m g \sin \left(-\Theta_{0}+\alpha_{0}\right)-D_{0}=0
$$

Normal to this direction,

$$
L_{0}+F_{0} \sin \alpha_{0}-m g \cos \left(-\Theta_{0}+\alpha_{0}\right)=0 .
$$

These equations can be solved for the descent angle $-\Theta_{0}+\alpha_{0}$ to give

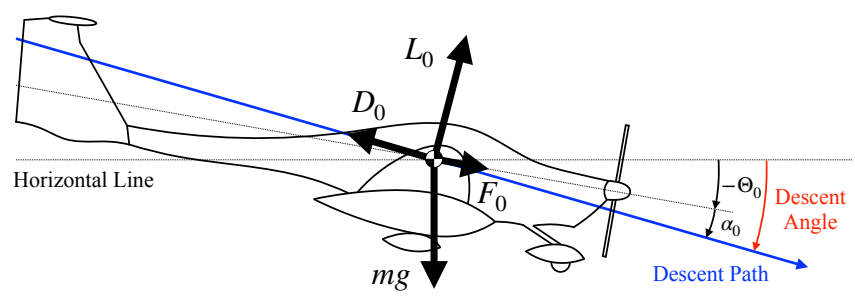

(a)

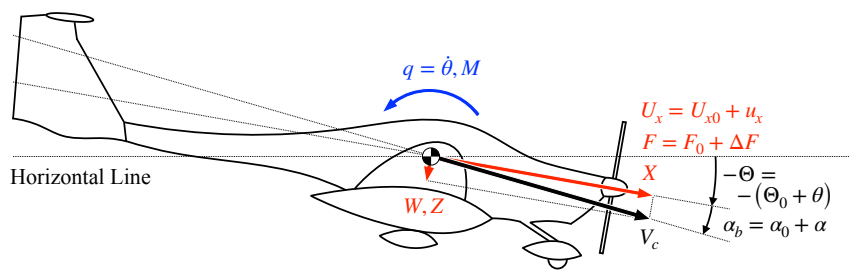

(b)

Fig. 2. (a) View of aircraft in steady descent. (b) Velocities, forces, and moments acting on descending aircraft.

$$
\tan \left(-\Theta_{0}+\alpha_{0}\right)=\frac{D_{0}-F_{0} \cos \alpha_{0}}{L_{0}+F_{0} \sin \alpha_{0}}
$$

$L_{0}$ and $D_{0}$ are given by the following equations.

$$
\begin{aligned}
L_{0} & =\frac{1}{2} \rho V^{2} S C_{L}\left(\alpha_{0}\right), \\
D_{0} & =\frac{1}{2} \rho V^{2} S C_{D}\left(\alpha_{0}\right) .
\end{aligned}
$$

Assuming that $\alpha_{0} \simeq 0$ and $L_{0} \simeq m g$, (3) becomes

$$
\tan \left(-\Theta_{0}+\alpha_{0}\right) \simeq \frac{D_{0}-F_{0}}{L_{0}} \simeq \frac{1}{L_{0} / D_{0}}-\frac{F_{0}}{m g} \ldots \ldots
$$

(6) suggests that the descent angle $-\Theta_{0}+\alpha_{0}$ is a function of lift-drag ratio $L_{0} / D_{0}$ and $F_{0}$. Conventional mechanical air brakes change $L_{0} / D_{0}$ to adjust the descent angle. Conversely, the proposed regenerative air brake changes $F_{0}$ to adjust the descent angle.

Fig. 2(b) shows the velocities, forces, and moment acting on descending aircraft. The linearized state-space equation of the vertical motion is shown in (7)-(11) ${ }^{(13)}$.

$$
\begin{aligned}
\dot{\boldsymbol{x}} & =\boldsymbol{A} \boldsymbol{x}+\boldsymbol{B u}, \cdots \cdots \cdots \\
\boldsymbol{x} & =\left[\begin{array}{llll}
u_{x} & \alpha & q & \theta
\end{array}\right]^{T} \\
\boldsymbol{u} & =\left[\begin{array}{lll}
\delta_{\mathrm{ab}} & \delta_{\mathrm{t}}
\end{array}\right]^{T}, \cdots \cdots
\end{aligned}
$$

The state variables $\boldsymbol{x}$ in (8) are the perturbed variables, which are the changes from the trim derived from (1) and (2). Let $\Delta F$ be the thrust change from the trim. Because the regenerative air brake uses only thrust $\Delta F$ to change the attitude, $\boldsymbol{u}$ and $\boldsymbol{B}$ become

$$
\begin{aligned}
u_{\mathrm{RA}} & =\Delta F, \cdots \ldots \ldots \ldots \ldots \\
\boldsymbol{B}_{\mathrm{RA}} & =\left[\begin{array}{llll}
\frac{1}{m} & 0 & 0 & 0
\end{array}\right]^{T} .
\end{aligned}
$$

Therefore, the transfer function from $\Delta F$ to $q$ is given as follows.

$$
\frac{q}{\Delta F}=\left[\begin{array}{llll}
0 & 0 & 1 & 0
\end{array}\right](s \boldsymbol{I}-\boldsymbol{A})^{-1}\left[\begin{array}{c}
\frac{1}{m} \\
0 \\
0 \\
0
\end{array}\right] \ldots \cdots
$$




$$
\begin{aligned}
& \boldsymbol{A}=\left[\begin{array}{cccc}
X_{u_{x}} & X_{\alpha} & X_{q}-W_{0} & -g \cos \Theta_{0} \\
Z_{u x} / U_{x 0} & Z_{\alpha} / U_{x 0} & Z_{q} / U_{x 0}+1 & -g \sin \Theta_{0} / U_{x 0} \\
M_{u_{x}}+M_{\dot{\alpha}}\left(Z_{u_{x}} / U_{x 0}\right) & M_{\alpha}+M_{\dot{\alpha}}\left(Z_{\alpha} / U_{x 0}\right) & M_{q}+M_{\dot{\alpha}}\left(Z_{q} / U_{x 0}+1\right) & -M_{\dot{\alpha}} g \sin \Theta_{0} / U_{x 0} \\
0 & 0 & 1 & 0
\end{array}\right], \\
& \boldsymbol{B}=\left[\begin{array}{cc}
X_{\delta_{\mathrm{ab}}} & X_{\delta_{\mathrm{t}}} \\
Z_{\delta_{\mathrm{ab}}} / U_{x 0} & Z_{\delta_{\mathrm{t}}} / U_{x 0} \\
M_{\delta_{\mathrm{ab}}}+M_{\dot{\alpha}}\left(Z_{\delta_{\mathrm{ab}}} / U_{x 0}\right) & M_{\delta_{\mathrm{t}}}+M_{\dot{\alpha}}\left(Z_{\delta_{\mathrm{t}}} / U_{x 0}\right) \\
0 & 0
\end{array}\right]
\end{aligned}
$$

$$
\begin{aligned}
& =B \int(\mathrm{d} L \cos \phi-\mathrm{d} D \sin \phi), \\
Q & =B \int \mathrm{d} Q, \cdots \cdots \cdots \cdots \cdots \cdots \cdots \cdots \\
& =B \int r(\mathrm{~d} L \sin \phi+\mathrm{d} D \cos \phi)
\end{aligned}
$$

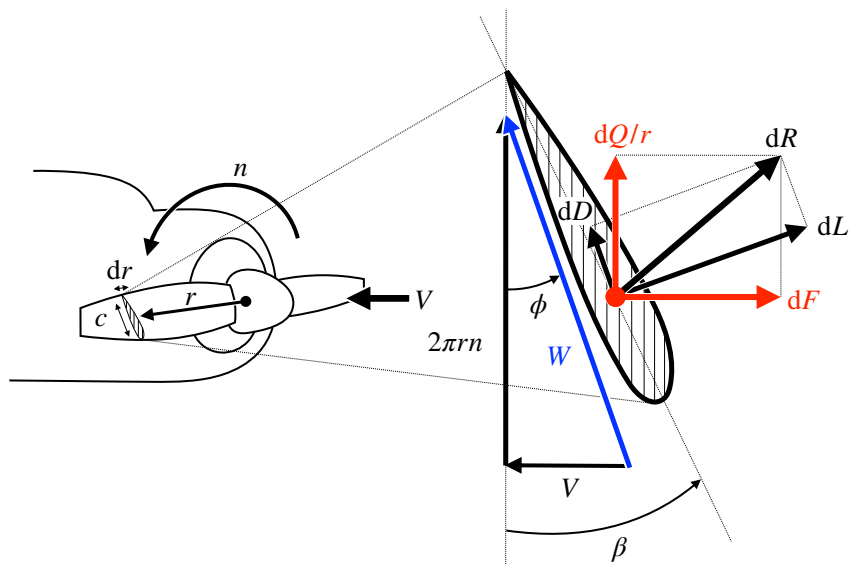

(a)
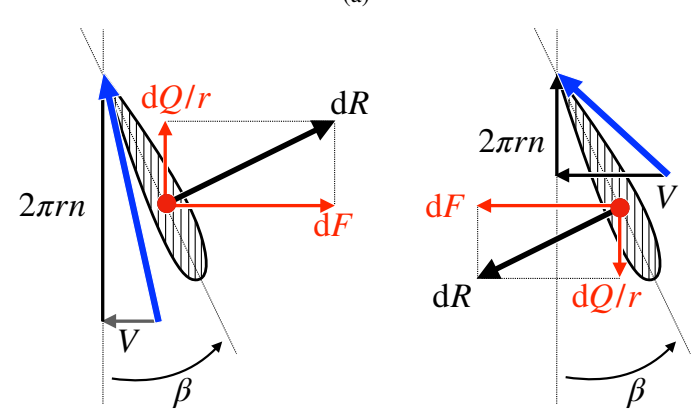

(b)

Fig. 3. (a) Velocities and forces acting on propeller blade element. (b) View of the powering propeller (left) and the windmilling propeller (right).

2.2 Propeller Dynamics A propeller-driven aircraft obtains all of its thrust from the propeller. Fig. 3(a) shows the velocities and forces acting on the propeller blade element. $\mathrm{d} L$ is the differential lift, and $\mathrm{d} D$ is the differential drag. The contributions of the blade element to $F$ and $Q$ are as follows.

$$
\begin{aligned}
\mathrm{d} F & =\mathrm{d} L \cos \phi-\mathrm{d} D \sin \phi, \\
\mathrm{d} Q / r & =\mathrm{d} L \sin \phi+\mathrm{d} D \cos \phi .
\end{aligned}
$$

Similar to (4) and (5), $\mathrm{d} L$, and $\mathrm{d} D$ can be calculated by

$$
\begin{aligned}
\mathrm{d} L & =\frac{1}{2} \rho W^{2} c \mathrm{~d} r C_{L}, \\
\mathrm{~d} D & =\frac{1}{2} \rho W^{2} c \mathrm{~d} r C_{D},
\end{aligned}
$$

where $c$ denotes the chord. Let $B$ be the number of blades; then,

$$
F=B \int \mathrm{d} F,
$$

Considering the theoretical equations (15)-(22), $C_{F}$ and $C_{Q}$ are defined from the experimental results as follows ${ }^{(13)}$.

$$
\begin{aligned}
& C_{F}=\frac{F}{\rho n^{2} D_{p}^{4}}, \\
& C_{Q}=\frac{Q}{\rho n^{2} D_{p}^{5}} .
\end{aligned}
$$

From Fig. 3(a), the angle of the resultant flow $\phi$ is determined by the ratio of $V$ to $2 \pi n r$.

$$
\tan \phi=\frac{V}{2 \pi n r}=\frac{J}{\pi \frac{2 r}{D_{p}}} .
$$

$J$ is called the advance ratio, and is defined by

$$
J=\frac{V}{n D_{p}}
$$

Thus, $C_{F}$ and $C_{Q}$ are functions of $J . F$ and $Q$ can be written as follows.

$$
\begin{aligned}
& F=C_{F}(J) \rho n^{2} D_{p}^{4}, \\
& Q=C_{Q}(J) \rho n^{2} D_{p}^{5} .
\end{aligned}
$$

Fig. 3(b) shows a view of the powering propeller (left) and windmilling propeller (right). As the figures show, if the directions of $F$ and $Q$ are positive when powering, the propeller in the windmilling state produces negative $F$ and $Q$. In other words, $C_{F}$ and $C_{Q}$ become negative at a high $J$.

The equation of motion of the electric motor is given by

$$
T-Q=2 \pi J_{\omega} \frac{\mathrm{d} n}{\mathrm{~d} t}+2 \pi B_{\omega} n+T_{C}
$$

Ignoring the friction of the motor in (29), the input power $P$ is calculated by

$$
P=2 \pi n T=2 \pi n Q .
$$

$P$ becomes negative when $Q$ is negative, which enables power regeneration. In addition, from (6), negative thrust yields a wider range of descent angles.

\section{Proposal of Pitch Angle Control by Regenera- tive Air Brake}

The proposed method comprises four steps. Step 1 is thrust estimation, Step 2 is negative thrust control, Step 3 is airspeed estimation, and Step 4 is pitch angle control. Fig. 4 shows the overall block diagram of the proposed method. 


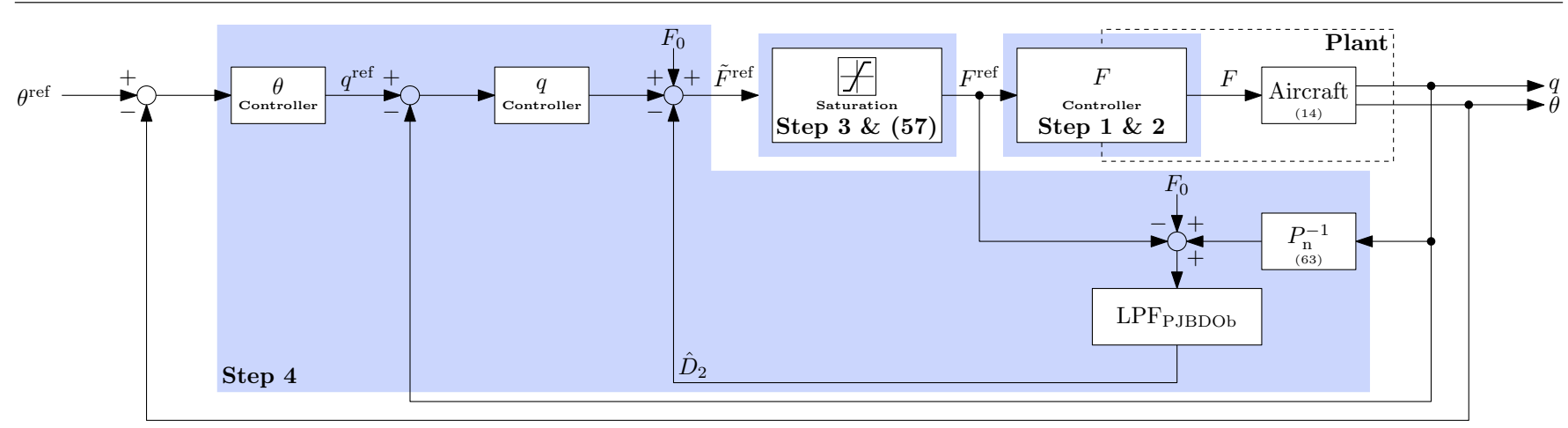

Fig. 4. Overall view of proposed method.

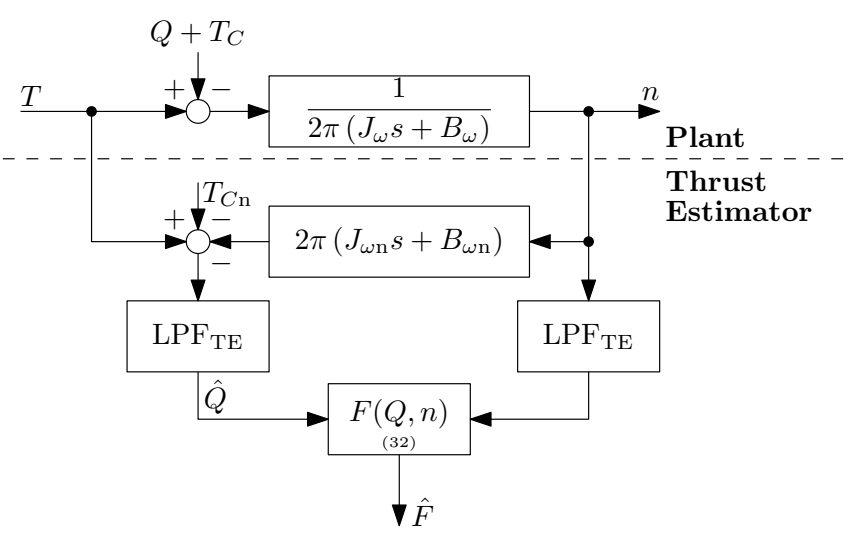

Fig. 5. Step 1: Thrust estimator (TE).

3.1 Step 1: Thrust Estimation To control the pitch angle, the thrust must be estimated because adding force sensors decreases the stiffness of the thrusters. In Step 1, the observer-based $^{(14)(15)}$ thrust estimator (TE) is proposed. A block diagram of the TE is shown in Fig. 5. $\mathrm{LPF}_{\mathrm{TE}}$ is a firstorder low-pass filter.

As shown in Fig. 3(a), $F$ and $Q$ are produced from the same aerodynamic force; therefore, it is reasonable to estimate $F$ from $Q . C_{F}(J)$ and $C_{Q}(J)$ are approximated using polynomials in conventional modeling. However, as observed in Fig. 6(a) and Fig. 6(b), the relation between $J$ and the coefficients have significant errors owing to the difference in the Reynolds number.

The proposed model uses the $C_{Q}-C_{F}$ relation. This model has two main advantages: (1) it has fewer errors owing to the difference in the Reynolds number, as shown in Fig. 6(c), and (2) $C_{Q}-C_{F}$ can be linearly approximated in both the powering and windmilling states, as shown in Fig. 6(c) and Fig. 6(d). Thus,

$$
\begin{aligned}
C_{F} & =a C_{Q}+b, \cdots \cdots \\
\hat{F} & =a \frac{\hat{Q}}{D_{p}}+b \rho n^{2} D_{p}^{4} .
\end{aligned}
$$

Regarding the APC propeller $11 \times 4$ used in the experiments, the maximum error of the conventional model is approximately $9 \%$, whereas that of the proposed model is approximately $4 \%$.

3.2 Step 2: Negative Thrust Control The negative thrust must be controlled to maintain a constant descent angle during the power regeneration. In Step 2, a negative thrust

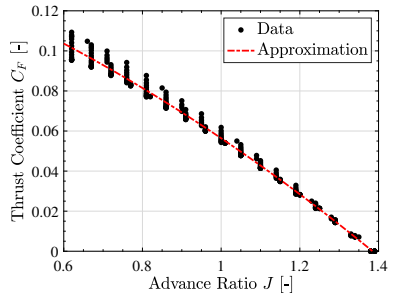

(a)

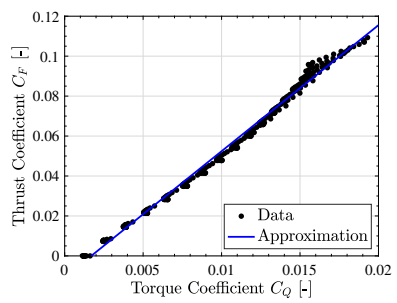

(c)

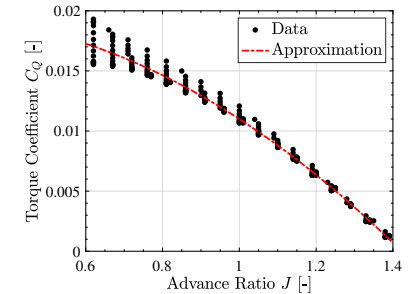

(b)

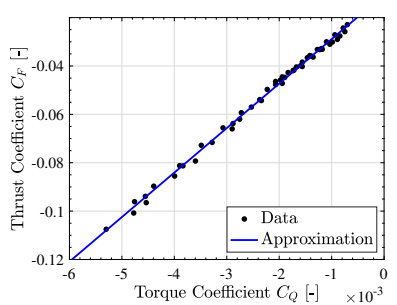

(d)
Fig. 6. (a) Relationship between $J$ and $C_{F}$ of APC propeller $10 \times 10^{(16)}$. (b) Relationship between $J$ and $C_{Q}$ of the APC propeller $10 \times 10^{(16)}$. (c) Relationship between $C_{Q}$ and $C_{F}$ of the APC propeller $10 \times 10^{(16)}$. (d) Relationship between $C_{Q}$ and $C_{F}$ of the windmilling APC propeller $11 \times 5.5^{(3)}$.

controller is proposed based on Step 1. Details of the controller design are provided in our previous paper ${ }^{(17)}$.

The negative thrust controller is designed as a two-degreeof-freedom controller and uses the estimated thrust $\hat{F}$ by the TE in the feedback controller. Fig. 7 shows the block diagram of the controller.

3.2.1 Rotational Speed Controller First, the rotational speed controller $C_{n}$ was designed. The rotational speed controller is a proportional controller that uses feedforward compensation by the disturbance observer (DOB), as shown in Fig. 8. In Fig. 8, the disturbance torque $T_{\mathrm{d}}$ is

$$
T_{\mathrm{d}}=Q+2 \pi B_{\omega} n+T_{C}
$$

The nominal plant is

$$
\frac{n}{T}=\frac{1}{2 \pi J_{\omega n} s} .
$$

$\mathrm{LPF}_{\mathrm{DOB}}$ is a first-order low-pass filter. The cut-off frequency should be higher than $\mathrm{LPF}_{\mathrm{TE}}$. The pole is placed at $\omega_{n}$.

3.2.2 Feedforward Controller Second, the feedforward controller $C_{\mathrm{FF}}$ was designed. Applying a quadratic approximation, $C_{F}(J)$ can be written as follows. 


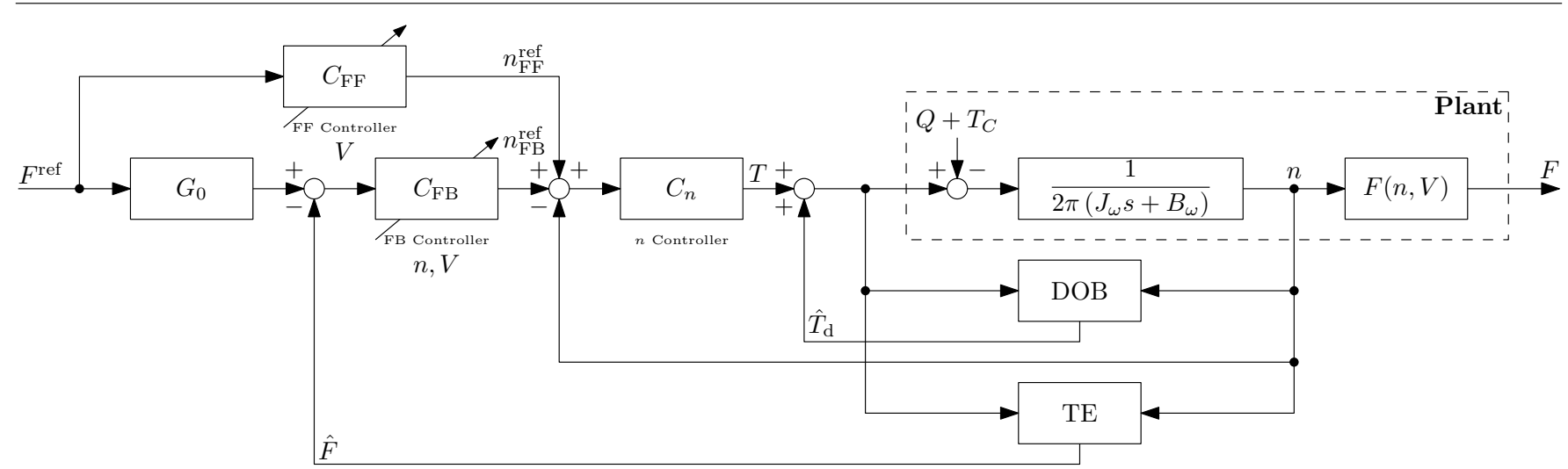

Fig. 7. Step 2: Negative thrust controller.

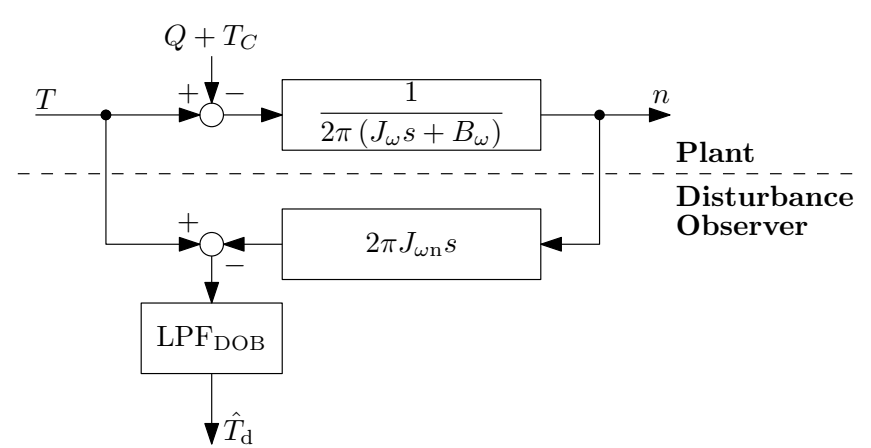

Fig. 8. Disturbance observer (DOB).

$$
C_{F}(J)=a_{C F} J^{2}+b_{C F} J+c_{C F}
$$

From (26), (27), and (35),

$$
\begin{aligned}
F & =\rho\left(a_{C F} V^{2} D_{p}^{2}+b_{C F} V D_{p}^{3} n+c_{C F} D_{p}^{4} n^{2}\right), \\
& =f(n) . \cdots \cdots \cdots \cdots \cdots \cdots \cdots \cdots \cdots \cdots \cdots \cdots \cdots \cdots \cdots
\end{aligned}
$$

From (37)

$$
F=f\left(\frac{n}{n^{\mathrm{ref}}} \cdot n^{\mathrm{ref}}\right)
$$

Thus, the feedfoward controller is given by

$$
n_{\mathrm{FF}}^{\mathrm{ref}}=\frac{s+\omega_{n}}{\omega_{n}} \cdot f^{-1}(F) \cdot G_{0}(s) .
$$

Filter $G_{0}(s)$ is a first-order low-pass filter with a cut-off frequency of $\omega_{\mathrm{FF}}$. The feedforward controller is a nonlinear variable controller because $f(n)$ is also a function of $V$.

3.2.3 Feedback Controller Finally, the feedback controller $C_{\mathrm{FB}}$ is designed. The Taylor expansion of (37) at the operating point $n=n_{0}$ gives the first-order approximation of $F$.

$$
F \approx a_{F}\left(n-n_{0}\right)+b_{F},
$$

where

$$
\begin{aligned}
& a_{F}=\rho\left(b_{C F} V D_{p}^{3}+2 c_{C F} D_{p}^{4} n_{0}\right), \ldots \ldots \ldots \ldots \\
& b_{F}=\rho\left(a_{C F} V^{2} D_{p}^{2}+b_{C F} V D_{p}^{3} n_{0}+c_{C F} D_{p}^{4} n_{0}^{2}\right) .
\end{aligned}
$$

The rotational speed controller is assumed to be sufficiently fast to obtain $n=n^{\text {ref }}$. The plant is assumed to be

$$
\frac{\Delta F}{\Delta n^{\mathrm{ref}}}=\frac{\Delta F}{\Delta n}=a_{F}
$$

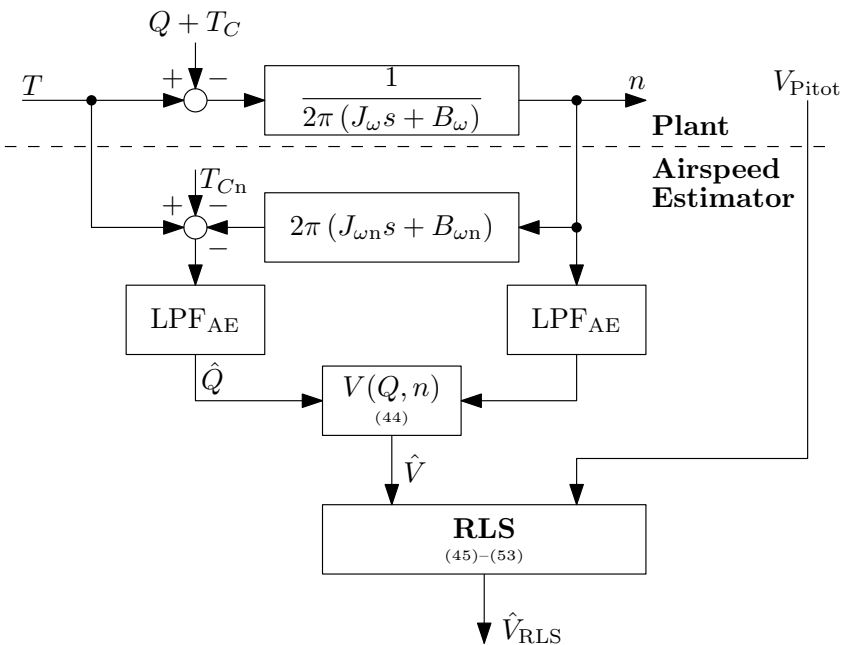

Fig. 9. Step 3: Airspeed estimator (AE).

The feedback controller $C_{\mathrm{FB}}$ is an integral controller because the plant is assumed to have no dynamics. In addition, it is a nonlinear variable controller because $a_{F}$ is a function of $n_{0}$ and $V$. The output of Step 1 was used as the feedback. The pole is placed at $\omega_{\mathrm{FB}}$.

3.3 Step 3: Airspeed Estimation The conditions of regeneration vary depending on the airspeed, but the conventional Pitot tube has poor responsiveness. The time constant of the Pitot tube on most aircraft is approximately a few seconds. To stay in the regenerative area, the airspeed must be estimated quickly. In Step 3, the observer-based airspeed estimation is performed by taking advantage of the accurate torque estimation from the current of the electric motors.

The idea of airspeed estimation using motor torque based on (28) was initially proposed by $\mathrm{JAXA}^{(18)}$; however, this method is sensitive to the modeling error shown in Fig. 6(b). In this study, an airspeed estimation method with real-time modification using a Pitot tube is proposed. In addition, the response times of the Pitot tube and the proposed method were compared.

A block diagram of the proposed AE is shown in Fig. 9. $\mathrm{LPF}_{\mathrm{AE}}$ is a first-order low-pass filter. Using (26), (28), and $\hat{Q}$, airspeed can be estimated as follows.

$$
\hat{V}=n D_{p} C_{Q}^{-1}\left(\frac{\hat{Q}}{\rho n^{2} D_{p}^{5}}\right)
$$


$\hat{V}$ captures rapid changes in airspeed, but its estimation accuracy depends on the modeling error shown in Fig. 6(b). Conversely, the measurement of the Pitot tube $V_{\text {Pitot }}$ has a slow response but high accuracy. Therefore, $\hat{V}$ is modified by $V_{\text {Pitot }}$ to achieve a quick and accurate estimation.

The coefficient $C_{V}$ defined by (45) is estimated using the recursive least-squares (RLS) method.

$$
V_{\text {Pitot }}=C_{V} \hat{V}
$$

In this estimation, the regression model is

$$
y=\varphi \theta .
$$

where output $y$, regressor $\varphi$, and estimation parameter $\theta$ are

$$
\begin{aligned}
y & =V_{\text {Pitot }}, \cdots \cdots \\
\varphi & =G_{\mathrm{RLS}}(s) \hat{V}, \\
\theta & =C_{V} . \cdots \cdots
\end{aligned}
$$

$G_{\mathrm{RLS}}(s)$ is designed to align the phases of $\hat{V}$ and $V_{\text {Pitot }}$ based on the fact that the response of the Pitot tube is theoretically modeled as a first-order delay ${ }^{(19)}$. Updates of these parameters are calculated as follows.

$$
\begin{aligned}
& \hat{\theta}[k]=\hat{\theta}[k-1]+\frac{P[k-1] \varphi[k]}{\lambda+P[k-1] \varphi^{2}[k]} \varepsilon[k], \cdots \\
& \varepsilon[k]=y[k]-\varphi[k] \hat{\theta}[k-1], \cdots \cdots \ldots \ldots \ldots \\
& P[k]=\frac{1}{\lambda}\left\{P[k-1]-\frac{P^{2}[k-1] \varphi^{2}[k]}{\lambda+P[k-1] \varphi^{2}[k]}\right\},
\end{aligned}
$$

where $\lambda$ is the forgetting factor. The forgetting factor is required to cope with the effects of time-varying parameters, such as crosswind and air density, and should be determined by monitoring the aerodynamic changes, such as temperature. Finally, the estimated value of airspeed $\hat{V}_{\mathrm{RLS}}[k]$ can be calculated by

$$
\hat{V}_{\mathrm{RLS}}[k]=\hat{\theta}[k] \hat{V}[k] .
$$

Note that the update of $\hat{\theta}$ is slow owing to $G_{\mathrm{RLS}}(s)$, but $\hat{V}_{\mathrm{RLS}}[k]$ can capture rapid changes because $\hat{V}$ is only filtered by $\mathrm{LPF}_{\mathrm{AE}}$.

The estimated airspeed $\hat{V}_{\text {RLS }}$ is used for the saturation before thrust control shown in Fig. 4 because a fast response saturation is necessary to prevent command values below the lower limit of the thrust. Fig. 10 shows the relationship between $n, V$, and $F$. Because the negative thrust has a lower limit depending on the airspeed, anti-windup must be considered. This is a critical problem for the regenerative air brake. Therefore, saturation is placed in the PJBDOb, as shown in Fig. 4. The calculation of the thrust lower limit $F_{\min }$ is as follows. From (37),

$$
\begin{aligned}
& \left.\frac{\partial f(n)}{\partial n}\right|_{n=n_{\min }}=0, \cdots \\
& \therefore n_{\min }=\frac{-b_{C F}}{2 c_{C F} D_{p}} V
\end{aligned}
$$

Then, $F_{\min }$ can be calculated using $\hat{V}_{\text {RLS }}$.

$$
\begin{aligned}
F_{\min } & =f\left(n_{\min }\right), \cdots \ldots \ldots \ldots \ldots \\
& =\rho D_{p}^{2} \hat{V}_{\mathrm{RLS}}^{2}\left(a_{C F}-\frac{b_{C F}^{2}}{4 c_{C F}}\right) .
\end{aligned}
$$

The upper limit of the thrust is determined by the specification of the motor.

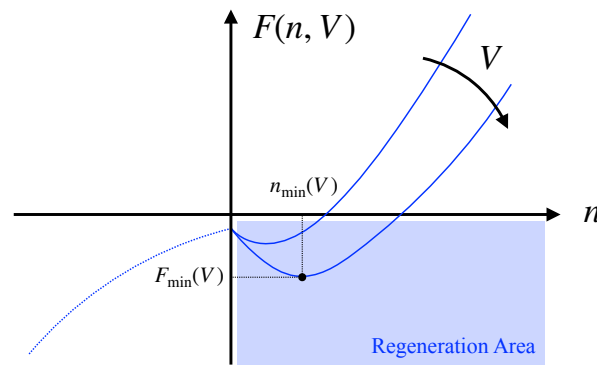

Fig. 10. Relationship between $n, V$, and $F$.

3.4 Step 4: Pitch Angle Control In this section, a pitch angle control method is proposed. This method is unique in that only thrust is used to control the pitch angle. Conventional aircraft use mechanical air brakes or elevators to adjust the descent path, and the thrust is not actively used to control the attitude. However, by taking advantage of electric motors, the EA achieves a quick thrust response. Therefore, the proposed negative thrust control enables not only power regeneration but also a high control performance of the attitude.

A block diagram of the pitch angle controller is shown in Fig. 4. This study focuses on pitch control, and an outer loop should be added to control the descent path. The proposed method comprises a pitch rate controller and a pitch angle controller.

3.4.1 Pitch Rate Controller The pitch rate controller is a proportional-derivative controller using a PJB$\mathrm{DOb}$, as shown in Fig. 4. LPF PJBDOb is a second-order lowpass filter. The output of the pitch-rate controller is transferred to the negative thrust controller.

From (7)-(14), the linearized equations of motion for $\Delta F$ and $q$ are

$$
\begin{aligned}
\dot{u}_{x}= & X_{u_{x}} u_{x}+X_{\alpha} \alpha+\left(X_{q}-W_{0}\right) q \\
& -g \cos \Theta_{0} \cdot \theta+\frac{1}{m} \Delta F, \ldots \ldots \ldots \ldots \ldots \ldots \\
\dot{q}= & \left(M_{u_{x}}+\frac{M_{\dot{\alpha}} Z_{u_{x}}}{U_{x 0}}\right) u_{x}+\left(M_{\alpha}+\frac{M_{\dot{\alpha}} Z_{\alpha}}{U_{x 0}}\right) \alpha \\
& +\left\{M_{q}+M_{\dot{\alpha}}\left(\frac{Z_{q}}{U_{x 0}}+1\right)\right\} q+\frac{-M_{\dot{\alpha}} g \sin \Theta_{0}}{U_{x 0}} \theta
\end{aligned}
$$

Because the regenerative air brake controls $\theta$ by the negative thrust, only the dynamics from $\Delta F$ to $q$ should be focused, and other terms are considered to be disturbance $D_{1}$. Thus,

$$
\left\{\frac{1}{m}\left(M_{u_{x}}+\frac{M_{\dot{\alpha}} Z_{u_{x}}}{U_{x 0}}\right)\right\}^{-1} \ddot{q}=\Delta F+D_{1}
$$

Similarly, using the nominal stability derivatives, we obtain

$$
\left\{\frac{1}{m_{\mathrm{n}}}\left(M_{u_{x} \mathrm{n}}+\frac{M_{\dot{\alpha} \mathrm{n}} Z_{u_{x} \mathrm{n}}}{U_{x 0 \mathrm{n}}}\right)\right\}^{-1} \ddot{q}=\Delta F+D_{2} . \cdots \cdots
$$

$D_{2}$ includes $D_{1}$ and modeling error.

$$
\begin{aligned}
D_{2}= & D_{1}+\left[\left\{\frac{1}{m_{\mathrm{n}}}\left(M_{u_{x} \mathrm{n}}+\frac{M_{\dot{\alpha} n} Z_{u_{x} \mathrm{n}}}{U_{x 0 \mathrm{n}}}\right)\right\}^{-1}\right. \\
& \left.-\left\{\frac{1}{m}\left(M_{u_{x}}+\frac{M_{\dot{\alpha}} Z_{u_{x}}}{U_{x 0}}\right)\right\}^{-1}\right] \ddot{q} . \cdots .
\end{aligned}
$$




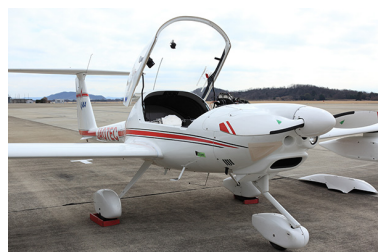

(a)

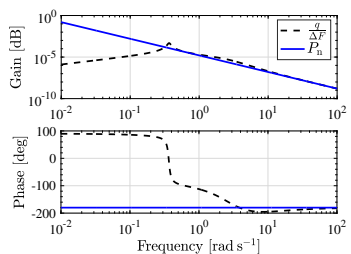

(b)

Fig. 11. (a) Picture of Diamond Aircraft HK-36 TTC $\mathrm{ECO}^{(21)}$. (b) Bode diagram of Diamond Aircraft HK-36 TTC ECO ${ }^{(22)}$.

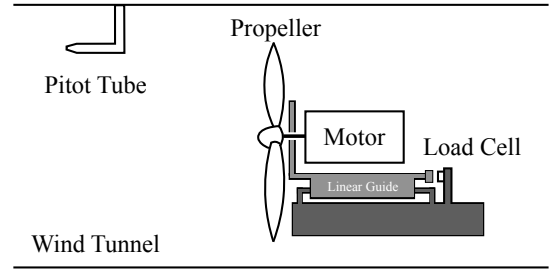

(a)

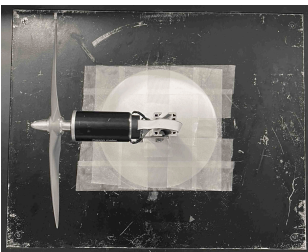

(b)

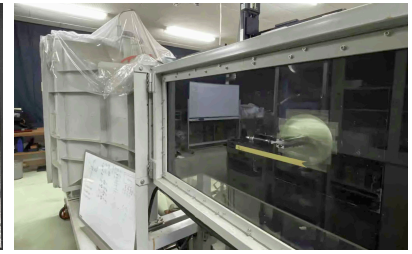

(c)
Fig. 12. (a) Experimental setup. (b) Picture of experimental setup. (c) Picture of wind tunnel.

PJBDOb estimates and compensates $D_{2}$, and plant $\frac{q}{\Delta F}$ is normalized to

$$
P_{\mathrm{n}}=\frac{1}{m_{\mathrm{n}}}\left(M_{u_{x} \mathrm{n}}+\frac{M_{\dot{\alpha} \mathrm{n}} Z_{u_{x} \mathrm{n}}}{U_{x 0 \mathrm{n}}}\right) \frac{1}{s^{2}}
$$

in the bandwidth of $\mathrm{LPF}_{\mathrm{PJBDOb}}{ }^{(20)}$. Based on the parameters of Diamond Aircraft HK-36 TTC ECO shown in Fig. 11(a) ${ }^{(21)}$, the bode diagrams of $\frac{q}{\Delta F}$ and $P_{\mathrm{n}}$ are shown in Fig. $11(\mathrm{~b})^{(22)}$. The multi-axis interference $D_{1}$ appears in the lowfrequency range, but this difference is compensated for in the target band.

The pole of the pitch rate controller is placed at $\omega_{q}$.

3.5 Pitch Angle Controller The pitch angle controller is a proportional controller. The pole is placed at $\omega_{\theta}$.

\section{Experiment and Simulation}

In this section, the wind tunnel experiments and simulations are described.

4.1 Setup Fig. 12 shows a diagram and pictures of the experimental setup. The experimental unit comprises a linear guide, load cell, motor, encoder, APC propeller $11 \times 4$, a Pitot tube, and a wind tunnel. The load cell measures $F$, the encoder measures $n$, and the Pitot tube measures $V$.

Before the experiment, $J_{\omega}, B_{\omega}$, and $T_{C}$ values were identified. As shown in Fig. 13(a), the motor torque was measured at constant speeds without the propeller, which determined $B_{\omega}$ and $T_{C}$. Thereafter, the torque was measured with the propeller at constant $n$ and $V=0 \mathrm{~m} \mathrm{~s}^{-1}$, as shown in Fig. 13(b). Because $V=0 \mathrm{~m} \mathrm{~s}^{-1}, C_{Q}$ is constant; therefore,

$$
Q \propto n^{2},
$$

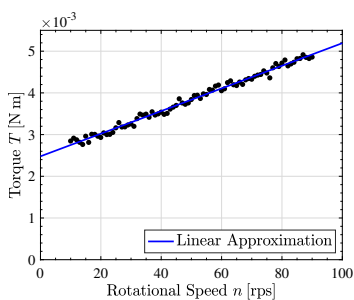

(a)

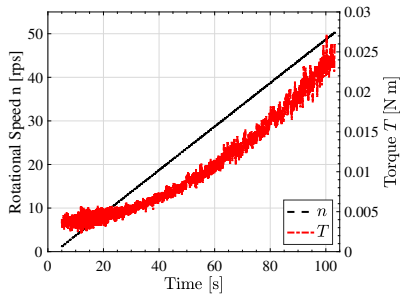

(c)

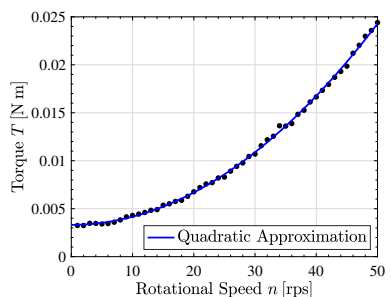

(b)

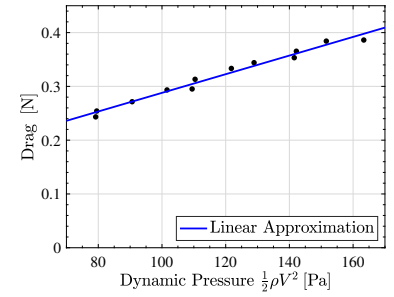

(d)
Fig. 13. (a) Measurement of $B_{\omega}$ and $T_{C}$. (b) $n-T$ relation with the propeller at a constant $n$. (c) Ramp response with the propeller. (d) Drag measurement of the experimental unit.

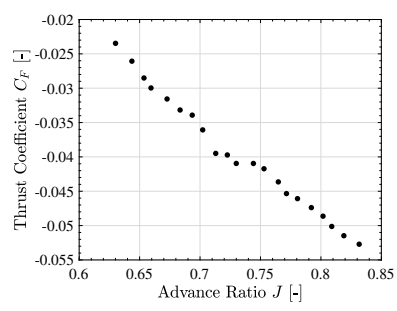

(a)

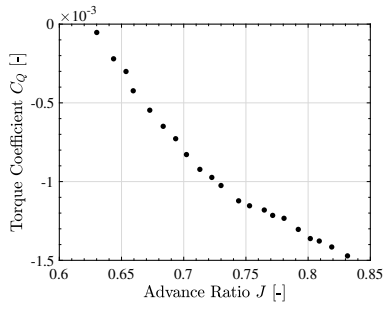

(b)
Fig. 14. (a) $C_{F}$ of test propeller. (b) $C_{Q}$ of test propeller.

Table 2. Experiment parameters.

\begin{tabular}{ll}
\hline Parameters & Value \\
\hline$J_{\omega}$ & $1.29 \times 10^{-4} \mathrm{~kg} \mathrm{~m}^{2}$ \\
$B_{\omega}$ & $4.32 \times 10^{-6} \mathrm{~N} \mathrm{~m} \mathrm{~s} \mathrm{rad}^{-1}$ \\
$T_{C}$ & $2.48 \times 10^{-3} \mathrm{~N} \mathrm{~m}$ \\
\hline
\end{tabular}

$$
T=\left(C_{Q} \rho D_{p}^{5}\right) n^{2}+\left(2 \pi B_{\omega}\right) n+T_{C} .
$$

Thus, a quadratic approximation is applied to the torque in Fig. 13(b). Finally, $J_{\omega}$ was calculated by measuring the torque with the propeller at $V=0 \mathrm{~m} \mathrm{~s}^{-1}$ under a constant acceleration command and by subtracting the result in Fig. 13(b), as shown in Fig. 13(c). Table 2 shows the parameters measured in the preliminary experiment.

Additionally, the drag on the experimental unit was measured and linearly approximated, assuming that the drag is proportional to the dynamic pressure $\frac{1}{2} \rho V^{2}$, as shown in Fig. 13(d). This drag was subtracted from the load-cell measurements in the experiments. The performance of the propeller was also tested, as shown in Fig. 14(a) and Fig. 14(b).

\subsection{Result}

4.2.1 Experimental Result of Step $1 \& 2$ In this experiment, $\omega_{n}=100 \mathrm{rad} \mathrm{s}^{-1}, \omega_{\mathrm{FF}}=50 \mathrm{rad} \mathrm{s}^{-1}, \omega_{\mathrm{FB}}=$ $50 \mathrm{rad} \mathrm{s}^{-1} . V$ is $14.8 \mathrm{~m} \mathrm{~s}^{-1}$.

Fig. 15(a) shows the step response of $F$, and Fig. 15(b) shows the time response of the rotational speed $n$. The thrust reference exponentially changed from $-0.82 \mathrm{~N}$ to $-1.32 \mathrm{~N}$ at 


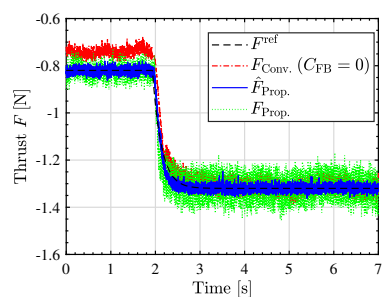

(a)

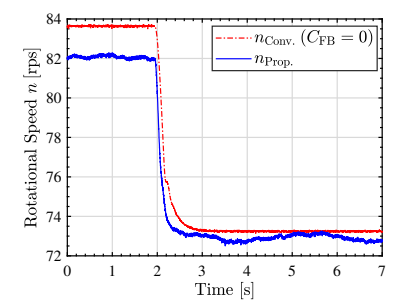

(b)
Fig. 15. (a) Step response of $F$. (b) $n$.

Table 3. Error comparison of experimental results.

\begin{tabular}{lll}
\hline Time & Thrust error of Conv. & Thrust error of Prop. \\
\hline$t=0-1 \mathrm{~s}$ & $9.51 \%$ & $1.95 \%$ \\
$t=5-6 \mathrm{~s}$ & $2.12 \%$ & $1.89 \%$ \\
\hline
\end{tabular}

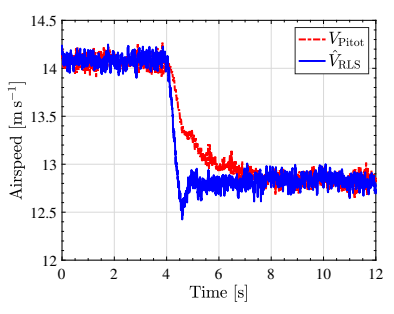

(a)

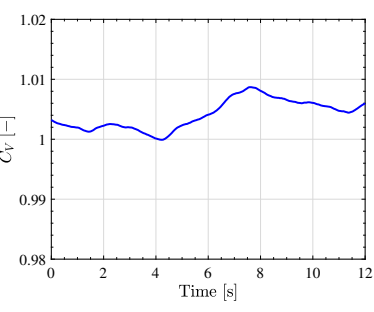

(b)
Fig. 16. (a) Step response of $V$. (b) $C_{V}$.

$t=2 \mathrm{~s} . \hat{F}_{\text {Prop. }}$ is the output of the TE, which is the controller feedback. $F_{\text {Prop. }}$ is the real value of the thrust measured by the load cell. For comparison, the experimental results of the conventional method $\left(C_{\mathrm{FB}}=0\right)$ is also shown in Fig. 15(a) and Fig. 15(b). Fig. 15(a) shows that the TE achieved accurate estimation, and the negative thrust controller achieved a quick response. A comparison of the thrust errors $\left|\frac{\bar{F}-\overline{F^{\text {ref }}}}{F_{\text {ref }}}\right|$ in the conventional and proposed methods is shown in Table 3. As shown in Table 3, the conventional method has larger errors than the proposed method because the conventional method uses only $C_{\mathrm{FF}}$, whereas the proposed method uses accurate TE for the feedback controller. The modeling error of the conventional method is large at high $J$, as observed in Table 3, which can also be observed in Fig. 6(a).

4.2.2 Experimental Result of Step 3 Fig. 16(a) shows the step response of $V$. The rotational speed of the wind tunnel fan was changed stepwise at $t=4 \mathrm{~s}$. $P[0]$ was 10,000 , and $\lambda$ was 0.99 because 100 samples of data were expected to capture the rapid changes in the environment. The time constant of the Pitot tube was $1.5 \mathrm{~s}$, and that of the proposed method was $0.3 \mathrm{~s}$. The proposed method achieved a five-fold faster estimation.

4.3 Simulation Result of Step 4 A simulation verified the proposed method using the parameters of the Diamond Aircraft HK-36 TTC ECO. These parameters were theoretically estimated from flight test results ${ }^{(23)}$. Details of the estimation are discussed in our previous paper ${ }^{(22)}$. Fig. 11(b) shows the bode diagram of $\frac{q}{\Delta F}$ obtained using the estimated parameter. The simulation considered a descending aircraft; therefore, $\Theta_{0}$ was set to $-3 \mathrm{deg}$ at $U_{x 0}=$ $3.00 \times 10^{1} \mathrm{~m} \mathrm{~s}^{-1}$ and $F_{0}=1.48 \times 10^{1} \mathrm{~N}$. In this simulation, $\omega_{q}=3 \mathrm{rad} \mathrm{s}^{-1}$ and $\omega_{\theta}=1 \mathrm{rad} \mathrm{s}^{-1}$. The cut-off frequency of

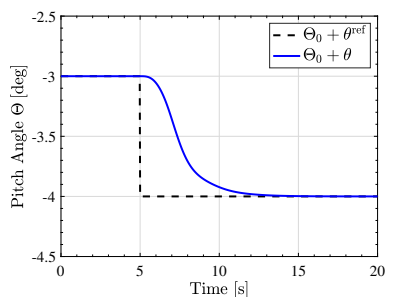

(a)

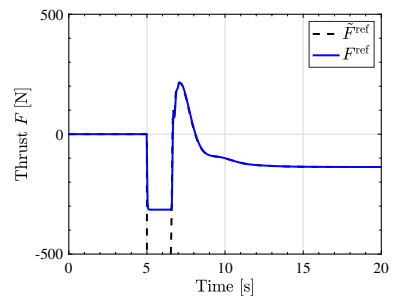

(c)

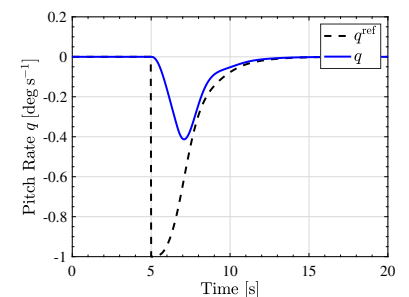

(b)

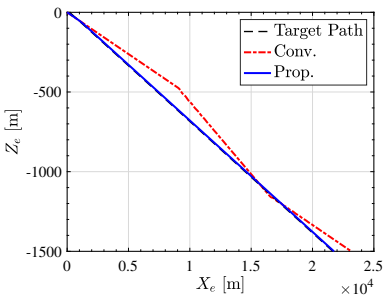

(d)
Fig. 17. (a) Step response of pitch angle. (b) Reference and output of pitch rate controller. (c) Control inputs $F_{1}$ and $F_{2}$. (d) Descent paths of conventional and proposed methods.

$\mathrm{LPF}_{\mathrm{PJBDOb}}$ was $50 \mathrm{rad} \mathrm{s}^{-1}$. The pole of the thrust controller was the same as that in Step 2 in this simulation.

The simulation results are shown in Fig. 17(a)-Fig. 17(d). Fig. 17(a) shows the step response of the pitch angle, Fig. 17(b) shows the reference and output of the pitch rate controller, and Fig. 17(c) shows the control input. As observed in Fig. 17(a), the response was quick enough for an aircraft. In addition, in Fig. 17(c), the control input was saturated, implying that the anti-windup controller was indispensable.

A comparison between the proposed method and conventional air brakes is shown in Fig. 17(d). Fig. 17(d) shows the descent paths of the proposed and conventional methods. $X_{e}$ and $Z_{e}$ are the earth-fixed coordinates. The proposed method uses a pitch angle controller, and the conventional method uses mechanical air brakes. The control derivatives of the mechanical air brakes were also estimated from the flight test results ${ }^{(23)}$. Mechanical air brakes change the airfoil and reduce $L / D$. When the mechanical air brakes are half-opened, $L / D$ decreases to 10.54 , and when fully opened, it decreases to 7.17. This change is demonstrated by the change in $F^{\text {ref }}$ for the negative thrust controller without the feedback of $q$ and $\theta$ as shown in Fig. 4.

Because mechanical air brakes do not have actuators, only open, half-opened, and closed types are available for use. Thus, in the simulation, the air brakes were half-opened when the aircraft was at a certain distance from the target path and closed when it was on the target path. Specifically, the mechanical air brakes were half-opened at $X_{e}=0.9 \mathrm{~m}$ and closed at $X_{e}=1.65 \mathrm{~m}$. With the conventional method, a descent angle of only $3 \mathrm{deg}$ or $5.2 \mathrm{deg}$ could be achieved; in contrast, the proposed method allowed a continuous change in the angle and enabled a descent at $4 \mathrm{deg}$.

These simulation results show that the proposed method takes advantage of the high control performance of electric motors and achieves better path tracking than conventional mechanical air brakes. 


\section{Conclusion}

Electric aircraft are safe, efficient, and environmentally friendly and are therefore attracting attention. Conventional aircraft use mechanical air brakes to adjust the descent angle, but their lift-drag ratio cannot be controlled continuously. In this paper, a pitch angle control method using the regenerative air brake is proposed. The proposed method includes thrust estimation, negative thrust control, airspeed estimation, and pitch angle control. Experiments and simulations verified the effectiveness of the proposed method.

\section{Acknowledgment}

The authors would like to thank Mr. H. Kobayashi from the Japan Aerospace Exploration Agency for his valuable advice and technical assistance with the experiments. This work was partly supported by JSPS KAKENHI (Grant Number JP18H03768).

\section{References}

( 1 ) Y. Hori, "Future Vehicle Driven by Electricity and Control-Research on Four-Wheel-Motored “UOT Electric March II ", IEEE Transactions on Industrial Electronics, vol. 51, no. 5, pp. 954-962, Oct 2004.

( 2 ) N. K. Borer, M. D. Patterson, J. K. Viken, M. D. Moore, J. Bevirt, A. M. Stoll, and A. R. Gibson, "Design and Performance of the NASA SCEPTOR Distributed Electric Propulsion Flight Demonstrator," in 16th AIAA Aviation Technology, Integration, and Operations Conference. AIAA, Jun 2016, pp. $1-20$.

( 3 ) N. Adachi, H. Kobayashi, H. Hakojima, and A. Nishizawa, "An Experimental Study on Energy Regeneration Using Propellers,” JAXA, Tech. Rep., 2015, (in Japanese).

( 4 ) K. Takahashi, H. Fujimoto, Y. Hori, H. Kobayashi, and A. Nishizawa, "Modeling of propeller electric airplane and thrust control using advantage of electric motor," in 2014 IEEE 13th International Workshop on Advanced Motion Control (AMC), no. 1. IEEE, Mar 2014, pp. 482-487.

( 5 ) N. Konishi, H. Fujimoto, Y. Watanabe, K. Suzuki, H. Kobayashi, and A. Nishizawa, "Lift control of electric airplanes by using propeller slipstream for safe landing," in 2015 IEEE International Conference on Mechatronics (ICM). IEEE, Mar 2015, pp. 335-340.

( 6 ) N. Konishi, H. Fujimoto, H. Kobayashi, and A. Nishizawa, "Range extension control system for electric airplane with multiple motors by optimization of thrust distribution considering propellers efficiency," in IECON 2014 - 40th Annual Conference of the IEEE Industrial Electronics Society. IEEE, Oct 2014, pp. 2847-2852.

( 7 ) H. Fujimoto and H. Sumiya, "Advanced safety range extension control system for electric vehicle with front- and rear-active steering and left- and right-force distribution," in 2012 IEEE/ASME International Conference on Advanced Intelligent Mechatronics (AIM). IEEE, Jul 2012, pp. 532-537.

( 8 ) Y. Xiang, H. Fujimoto, Y. Hori, Y. Watanabe, and K. Suzuki, "Proposal of Regeneration Power Control System by Optimization of Propeller Pitch Angle and Revolution Speed for Electric Airplanes," in IEE of Japan Technical Meeting Record, no. MEC-15-49, 2015, pp. 121-126, (in Japanese)

( 9 ) M. Okuyama, H. Nishizawa, and A. Kobayashi, "Aerodynamic Characteristics of Windmilling on Propeller," JAXA, Tech. Rep., 2015, (in Japanese)

(10) A. Nishizawa, H. Kobayashi, and H. Fujimoto, "Development and flight demonstration of regenerative electric propulsion system for aircraft application," in Electric E Hybrid Aerospace Technology Symposium, vol. 1, no. 4, 2015.

(11) T. Nodama and S. Sunada, "An Investigation on Battery Charging with a Propeller," Journal of the Japan Society for Aeronautical and Space Sciences, vol. 63, no. 1, pp. 8-12, 2015, (in Japanese).

(12) Y. Hayashi, D. Yashiro, K. Yubai, and S. Komada, "Experimental Validation of Contact Force Control of Quadrotor Based on Rotor Angular Acceleration Control," in 2019 IEEE International Conference on Mechatronics (ICM), vol. 1, no. 1. IEEE, Mar 2019, pp. 684-689.

(13) B. W. McCormick, Aerodynamics, Aeronautics and Flight Mechanics, 2nd ed. John Wiley \& Sons, Inc., 1995.

(14) S. Nagai, R. Oboe, T. Shimono, and A. Kawamura, "Fast Force Control with- out Force Sensor Using Combination of aaKF and RFOB for In-circuit Test with Probing System," IEEJ Journal of Industry Applications, vol. 8, no. 2, pp. 152-159, Mar 2019.

(15) S. Yamada and H. Fujimoto, "Minimum-Variance Load-Side External Torque Estimation Robust Against Modeling and Measurement Errors," IEEJ Journal of Industry Applications, vol. 9, no. 2, pp. 117-124, Mar 2020.

(16) APC, “APC Propellers Peformance Data." [Online]. Available: https://www.apcprop.com/technical-information/performance-data/

(17) K. Yokota, H. Fujimoto, and Y. Hori, "Basic Study on Regenerative Air Brake Using Observer-based Thrust Control for Electric Airplane," in 2020 IEEE 16th International Workshop on Advanced Motion Control (AMC). IEEE, Sep 2020, pp. 34-39.

(18) H. Kobayahi, A. Nishizawa, and T. Iijima, "Airspeed estimation by electric propulsion system parameters," in 55th Aircraft Symposium. JSASS, 2017, (in Japanese).

(19) R. P. Benedict, Fundamentals of Temperature, Pressure, and Flow Measurements. Wiley-Interscience, 1984.

(20) K. Ohnishi, M. Shibata, and T. Murakami, "Motion control for advanced mechatronics," IEEE/ASME Transactions on Mechatronics, vol. 1, no. 1, pp. 56-67, Mar 1996.

(21) JAXA, "Electric and hybrid propulsion system for aircraft." [Online]. Available: http://www.aero.jaxa.jp/eng/research/frontier/feather/

(22) K. Yokota, H. Fujimoto, and Y. Hori, "Descent Angle Control by Regenerative Air Brake Using Observer-based Thrust Control for Electric Aircraft," in AIAA Propulsion and Energy 2020 Forum. AIAA, Aug 2020, pp. 1-13.

(23) H. Kobayashi, T. Iijima, and A. Nishizawa, "Required Specifications of an Electric Propulsion System Based on Flight Test Using a Motor Glider," JAXA, Tech. Rep., 2015, (in Japanese).

Kentaro Yokota (Student Member) received a B.E. degree in the De-

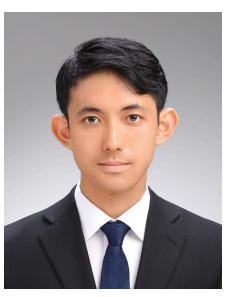
partment of Electrical and Electronic Engineering at the University of Tokyo. He is currently working towards his M.S. degree at the Department of Advanced Energy, Graduate School of Frontier Sciences, the University of Tokyo. His research interests include electric aircraft control. He is a student member of the Institute of Electrical Engineers of Japan, the Japan Society for Aeronautical and Space Sciences, the Institute of Electrical and Electronics Engineers, and the American Institute of Aeronautics and Astronautics.

Hiroshi Fujimoto (Senior Member) received a Ph.D. degree in the

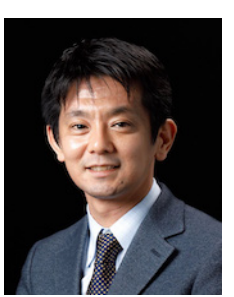
Department of Electrical Engineering from the University of Tokyo in 2001. In 2001, he joined the Department of Electrical Engineering, Nagaoka University of Technology, Niigata, Japan, as a research associate. From 2002 to 2003, he was a visiting scholar in the School of Mechanical Engineering, Purdue University, U.S.A. In 2004, he joined the Department of Electrical and Computer Engineering, Yokohama National University, Yokohama, Japan, as a lecturer and became an associate professor in 2005. He moved to the University of Tokyo as an associate professor in 2010 and became a professor in 2021. He received the Best Paper Awards from the IEEE Transactions on Industrial Electronics in 2001 and 2013, Isao Takahashi Power Electronics Award in 2010, Best Author Prize of SICE in 2010, the Nagamori Grand Award in 2016, and the First Prize Paper Award for IEEE Transactions on Power Electronics in 2016. His research interests include control engineering, motion control, nanoscale servo systems, electric vehicle control, motor drive, visual servo, and wireless motors. He is a senior member of the IEE of Japan and the IEEE. He is also a member of the Society of Instrument and Control Engineers, the Robotics Society of Japan, and the Society of Automotive Engineers of Japan. He was an associate editor of IEEE/ASME Transactions on Mechatronics from 2010 to 2014, IEEE Industrial Electronics Magazine from 2006, IEE of Japan Transactions on Industrial Application from 2013, and Transactions on SICE from 2013 to 2016. He is a chairperson of the JSAE vehicle electrification committee since 2014 and a past chairperson of IEEE/IES Technical Committee on Motion Control from 2012 to 2013. 